\title{
Le voile étanche métallique du barrage Salazar
}

\section{The watertight metal diaphragm in Salazar dam}

\author{
pAR N. FRANCO \\ INGÉNIEUR AUX ÉTABLISSEMENTS NEYRPIC \\ DÉTACHÉ AUPRÈS DES SOCIEDADES REUNIDAS DE FABRICAGOES METALLICAS
}

\begin{abstract}
L'utilisation d'un voile métallique pour l'étanchéité des barrages en terre ou en enrochement n'est pas une nouveauté mais a pu être négligée parfois. Ce procédé présente cependant de nontbreux avantages : étanchéité dans la masse; homogénéité; vitesse d'installation; simplification des travaux de remblayage; rapidité de mise en eall; souplesse; facilité de réparation.

Ces qualites, jointes au prix propre qui n'est déja pas plus élevé que celui d'autres solutions comparables, en rendent l'application très viable et économique.

lApres avoir donné des idées générales sur les voiles étanches métalliques, l'article décrit celui du barrage Salazar (Portugal) dont la surface est supérieare d̀ un hectare.

Le processus de montage et les engins spéciaux utilisés forment un chapitre essentiel.

Enfin la mise en service el la tenue du voile permettent de tirer des conclusions tout $\dot{a}$ fait favorables à l'utilisation du procédé.
\end{abstract}

\begin{abstract}
There is nothing new in using a watertight metal diaphragm for earth and rockfill dams but the method has been somewhat neglected. It does, howeser, have many advantages :

Complete watertightness; homogeneity; simpler placing of fill; quicker initial filling of dam; flexibility; ease of repair.

These qualities combined with its cost, which is not higher than that of other methods, make it worthwhile and economical.

After a general description of watertight metal diaphragms the author goes on to describe the one used in the Salazar Dam and gives details of its construction and of how it was protected from corrosion. The rea of its watertight diaphragm is more than one hectare.

The assembly procedure and the special handling equipment used are also described.

The performance of the diaphragm after it was put into service demonstrates the efficiency of the method.
\end{abstract}

\section{A. - DESCRIPTION D'ENSEMBLE DE L'OUVRAGE}

Parmi les nombreux et vastes travaux d'hydraulique agricole entrepris, ces dernières années, au Portugal, une place de choix revient à l'aménagement du cours inférieur du Sado, en vue de l'irrigation des terres de riz dont le rendement à l'hectare est très élevé.

Les 9613 hectares en question sont compris cntre les rivières Sado, Xarrama et le ruisseau de Santa Catarina (el desservis par $126 \mathrm{~km}$ de canaux). Ces deux derniers furent équipés de barrages. Le barrage Trigo de Morais, édifié sur' le premier au lieu dit Vale de Gaio, rappelle l'infatigable animateur de ces travaux, et le second, à Pego do Altar, a été nommé, d'après le chef du gouvernement portugais, barrage Salazar. L'un et l'autre de ces ouvrages sont les barrages en emrochement ou mixte les plus hauts construits jusqu'ici en Europe.

Le barrage Trigo de Morais, de type mixte enrochement et terre, a une hauteur de $51 \mathrm{~m}$ au-dessus des fondations et une retenue utile de 63 millions de $\mathrm{m}^{3}$, Il est muni d'un voile en béton asphaltique et protégé à l'amont par un vaste volume de terre.

Le barrage Salazar, qui nous concerne plus directement, a une hauteur de $63 \mathrm{~m}$ au-dessus de ses fondations et emmagasine 94 millions de $\mathrm{m}^{3}$ utiles. Il est muni d'un voile métallique étanche, objet du présent article.

En plus de l'irrigation et la régularisation des crues, ces ouvrages, comme la plupart des autres de la J.A.O.H.A., sont prévus pour fournir de l'énergie électrique, grâce à de petites centrales de pied de barrage. Celles du Sado, avec un total de $4130 \mathrm{ch}$ installés, permettent de livrer au réseau portugais environ 10 millions de kWh d'énergie d'été, puisque, sauf au moment de crues, les centrales ne sont mises en marche qu'aux époques d'irrigation. 
Quelques chiffres donneront un aperçu des dimensions et dispositions de l'ouvrage :

-.. Surface du bassin hydrogra-

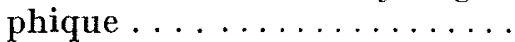

- Pluviométrie pondérée......

- Cote du couronnement......

- Cote du point le plus bas des fondations ............

- Largeur du couronnement...

- Longueur du couronnement. .

.. Talus amont . . . . . . . . . .

- Talus aval . . . . . . . . . .

.. Largeur à la base. . . . . . . . . . .

- Volume du barrage..........

- Poids . . . . . . . . . . . . .
$743 \mathrm{~km}^{2}$

$560 \mathrm{~mm}$

$56 \mathrm{~m}$

$-6,76 \mathrm{~m}$

$5 \mathrm{~m}$

$192 \mathrm{~m}$

$1,25 / 1$

$1,41 / 1$

$176 \mathrm{~m}$

$371113 \mathrm{~m}^{3}$

$648.672 t$
Le terrain de fondation était assez quelconque, constitué par des schistes devoniens, fracturés, veinés, semés de poches de quartz. De là le choix d'un type de barrage de longueur suffisamment ample pour répartir convenablement les charges. Ces difficultés de fondations ont encore été accrues par une large faille transversale où les travaux de fondation ont atteint localement la cote - 26 .

Le barrage est muni d'une galerie de visite courant tout le long de sa base et formant drain.

Le corps du barrage est constitué de blocs relativement gros pesant jusqu'à 8 ou 9 tonnes et la proportion des vides $\mathrm{y}$ atteint $35,5 \%$. A l'amont, une couche de maçonnerie sèche à $30,5 \%$ de vides forme support pour le voile étanche et a environ $2 \mathrm{~m}$ d'épaisseur à la base contre $0,8 \mathrm{~m}$ au couronnement.

Enfin, une couche de béton maigre d'une épaisseur moyenne de $0,20 \mathrm{~m}$ a servi à lisser le parement amont en vue du montage du voile.

Pendant la construction, les tassements maximums vérifiés ont été de $0,76 \mathrm{~m}$ à l'aval et $0,25 \mathrm{~m}$ à l'amont. En vue de tassements ultérieurs, les précautions suivantes ont été prises :

a) Surélévation maximum $0,60 \mathrm{~m}$,

b) Convexité du couronnement avec flèche de 0,5 vers l'amont,

c) Profil anont concave.

\section{B. - LE REVETEMENT METALLIQUE DES BARRAGES EN ENROCHEMENT}

Un examen, même superficiel, des solutions adoptées pour l'étanchéité des barrages en enrochement montre que celles-ci sont de types très différents, tant pour des raisons techniques que pour des raisons économiques.

Le matériau idéal doit être, non seulement intrinsèquement étanche, mais encore résistant et flexible pour se prêter aux déformations correspondant aux tassements inévitables dans des ouvrages de ce genre, ainsi qu'aux effets des variations thermiques éventuelles.

Si le noyau en argile domine pour les petits et moyens barrages, dès l'instant que l'on en I rouve dans les environs du chantier, et si les voiles en béton armé sont largement utilisés (ne citant que pour mémoire les platelages en bois), ces types de construction présentent des désavantages certains.

Le noyau en argile convenablement compacté s'est révélé d'une excellente étanchéité et, se trouvant bien protégé, constamment humide, et a l'abri des variations thermiques, ne nécessite pas de dispositions compliquées. Toutefois, la logique et l'économie indiquent toutes deux que la place de l'étanchéité est à l'amont, en contact avec l'eau. Ainsi la totalité du remblayage parlicipe à la résistance de l'ouvrage et le voile est accessible pour la surveillance et les réparations éventuelles.
Le voile en béton armé est utilisé depuis une quarantaine d'années. Pour être suffisamment étanche, il lui faut un minimum d'épaisseur, et, pour être raisonnablement rigide, il lui faut un minimum d'armatures, sans prétendre pour autant pouvoir résister à des tassements extrêmement sévères. Par ailleurs, formant de vastes surfaces exposées aux changements de température, il lui faut, soit posséder une résistance propre très considérable lui permettant de résister, sans se fendre, aux efforts développés, soit être muni de joints de dilatation éliminant ces efforts mais rompant l'homogénéité du voile et introduisent les risques correspondant à ces nombreuses liaisons.

Le voile métallique, lui, possède à un haut degré toutes les qualités requises. Tout d'abord, le matériau est, par lui-mềme et sans limitation de l'épaisseur, parfaitement imperméable. Ensuite il possède une résistance propre et une déformabilité largement supérieure à tous les autres. De plus, grâce à la soudure, l'introduction de joints de dilatation, raidisseurs, etc., ne rompt pas l'homogénéité du voile. Enfin, se trouvant sur le parement amont, la visite et l'évenluelle répartition - par simple soudure - sont non seulement possibles, mais faciles et rapides. Le voilc métallique convient particulièrement bien à des réservoirs saisonniers et en outre permet une vidange rapide de la retenue. 
Ce type d'ćtanchéité présente donc de très gros avantages techniques sur ceux qui peuvent lui être opposés - avantages techniques se cristallisant en une idée qui ne peut qu'être une préoccupation dominante de ceux qui conçoivent et exploitent les ouvrages : la sécurité. En effet, la solution métallique marque des points sur tous les chapitres, étanchéité, résistance, adaptabilité, el même réparations el contròle, sans parler de la mise en place.

Un autre facteur, également intéressant, semble militer en faveur du voile métallique : le facteur économique. Comme indiqué ci-dessus, le noyau en argile a besoin d'être soutenu par un contrc-barrage à l'amont. Dès que les dimensions de l'ouvrage augmentent, le coût de ce contre-barrage vient grever celui de l'enrochement utile d'une fraction importante, pouvant atteindre près de la moitić du prix total de construction, puisque les volumes à l'amont et à l'aval du noyau sont sensiblement égaux. Le voile amont en béton ne présente pas cet inconvénient fondamental. Par contre, il est nettement plus coûleux, la différence de prix à résistance ígale atteignant près de $70 \%$ selon des éva- luations américaines. A noter également la possibilité de monter le voile métallique simultanément avec le remblayage auquel il sert de coffrage, le barrage étant immédiatement utilisable presque jusqu'à la hauteur revêtue à n'importe quel moment de la construction.

Le voile étanche - voire même le barrage métallique - ne constituent pas des innovations. Dès la fin du siècle dernier, des barrages entièrement métalliques atteignant une trentaine de mètres de hauteur et constitués par des séries de chevalets en treillis réunis par des tôles légèrement cintrées et dont la concavité était tournce vers l'amont, furent construits aux Etats-Unis. Depuis, de nombreux revêtements, avec des caractéristiques diverses, ont été installés tant aux Etats-Unis qu'en Italie, en Géorgie, etc. Ce mode de construction se développait rapidement et n'eût été la guerre avec la pénurie de matériaux ferreux qu'elle a entraînée, aurait pris une extension bien plus considérable. Par ailleurs, les ingénieurs chargés du génie civil oublient trop souvent les possibilités que leur offre la construction métallique et le mariage des deux lechniques en a sans doute souffert.
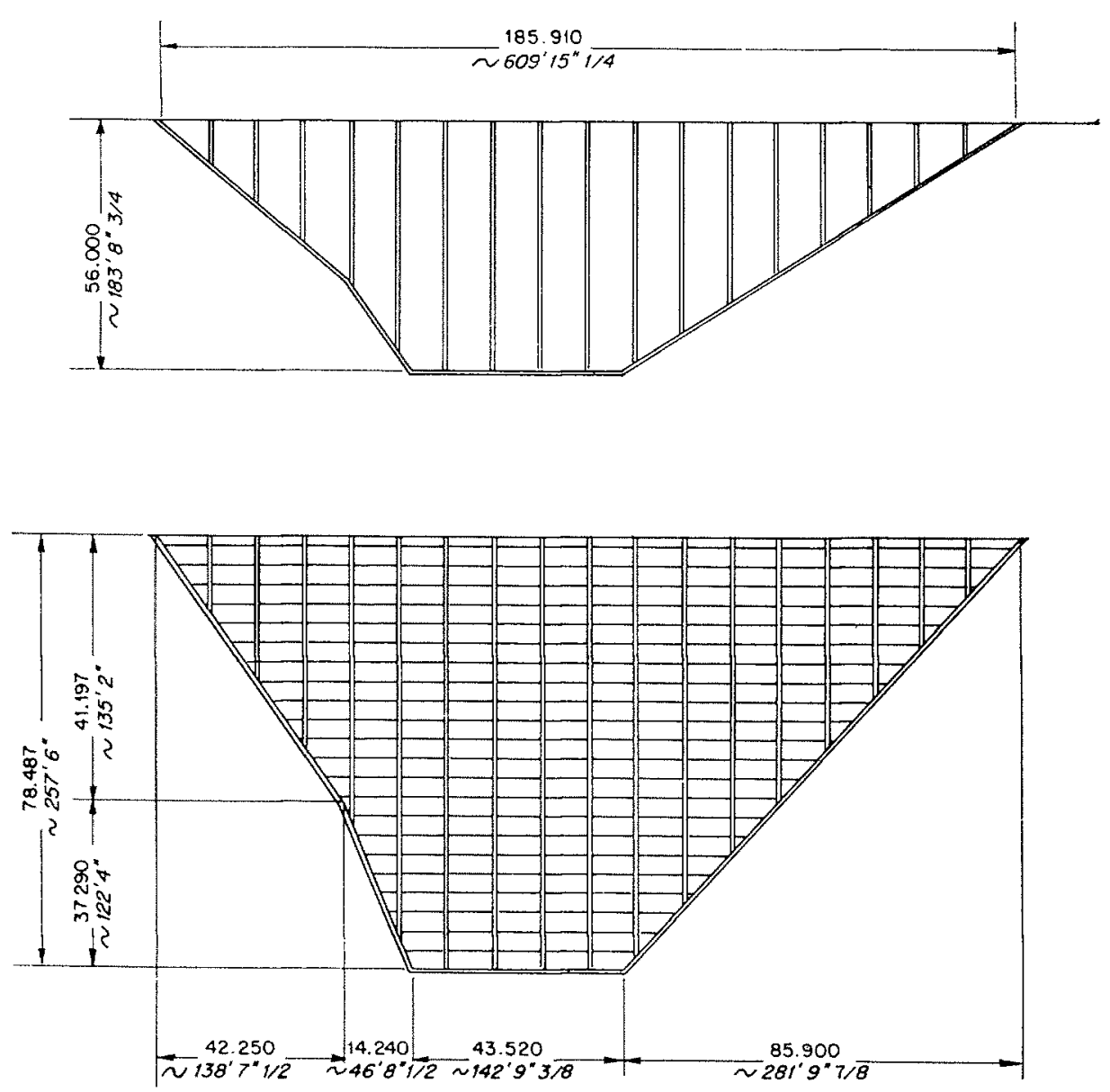

FIG. 1 


\section{C. - APPLICATION D'UN VOILE METAlLIQUe AU BARRAGE SALAZAR}

Le voile élanche du barrage Salazar a été installé après achèvement des travaux d'enrochement. Auparavant, comme il a été dit plus haut, le parement amont avait été muni d'une forte couche de maconnerie rangée et d'une chape de béton maigre destinée à rendre la surface suffisamment lisse.

Le type de voile choisi par l'administration a été celui à contraction libre dans les deux sens. Horizontalement, la contraction est absorbée par des joints flexibles, et, verticalement, par le déplacement d'ensemble $d u$ voile, et en particulier du bord supérieur. Toutes les liaisons sont réalisées par soudure, offrant ainsi toutes garanties quant à la résistance et à l'étanchéité.

Le matériau employé a été l'acier SiemensMartin doux selon ASTM-A-7 au cuivre, ayant une résistance à la traction de $42-50 \mathrm{~kg} / \mathrm{mm}^{2}$ et un allongement de $26 \%$. Sa composition chimique moyenne étant :

$\begin{array}{ll}\mathrm{C} & 0,2 \% \\ \mathrm{Mn} & 0,47 \% \\ \mathrm{P} & 0,019 \% \\ \mathrm{~S} & 0,028 \% \\ \mathrm{Cu} & 0,28 \%\end{array}$

La présence du cuivre étant prévue pour diminuer les effets de la corrosion.

L'épaisseur des tôles a été de $5 / 16^{\prime \prime}(7,937 \mathrm{~mm})$ pour le tiers inférieur et de $1 / 4^{\prime \prime}(6,35 \mathrm{~mm})$ pour Ia partie supérieure. Leurs dimensions étaient de $7,5 \mathrm{~m} \times 2,5 \mathrm{~m}$, avec le côté le plus long disposé horizontalement; ces côtés sont soudés bout à bout sur couvre-joint de $25 \times 3$ avec chanfreins à $45^{\circ}$ sur le bord supérieur; dans ces conditions, il est normalement admis que la soudure développe la pleine résistance de la tôle. Dans l'autre sens, les files de tôles sont limitées par des joints de dilatation -- contraction en demi-cercle conme indiqué figure 2.

Cette disposition a été adoptée de préférence à la forme de la figure 3 , celle-ci soumettant le sommet du joint à des flexions alternées sévères. Par ailleurs, les joints forment drains, recueillant les fuites éventuelles et les conduisant jusqu'à la galerie de drainage. En plus de la rigidité apportée par les joints de dilatation, les tôles sont raidies en leur milieu par des barres de $50 \times 8$ soudées sur champ et effilées à chaque bout vers le centre des tôles représentées figure 4 .
Chaque tôle est ancrée au barrage en son centre, sans pour cela créer des points fixes, par des tiges de scellement, de $1^{\prime \prime}$ de diamètre, et de

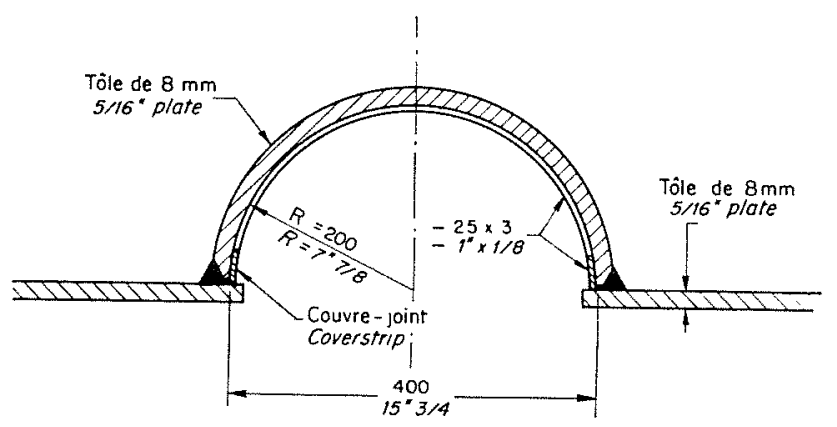

Fli. 2

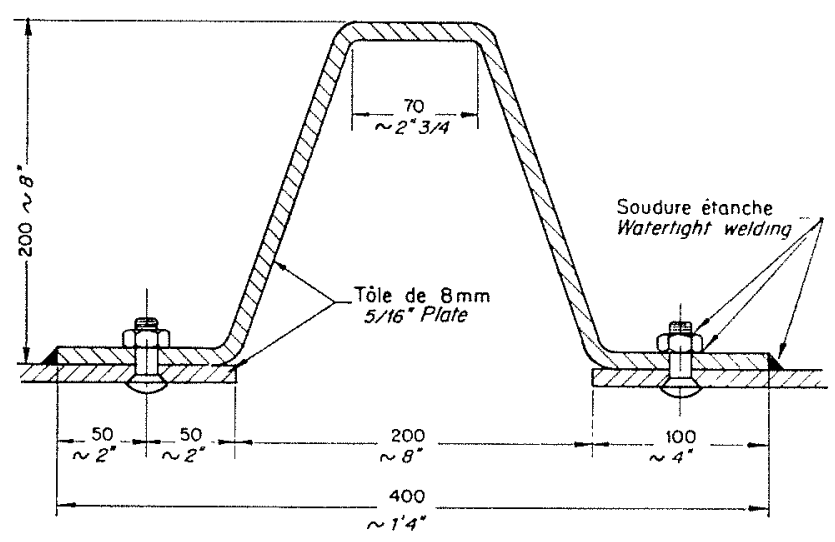

FIG. 3

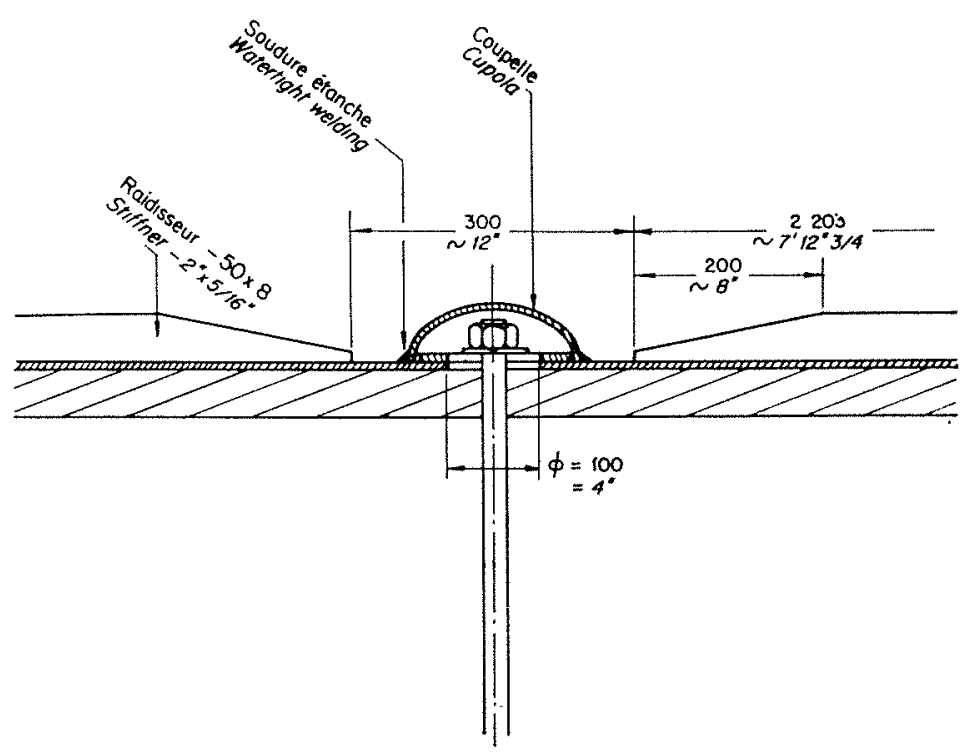

Fig. 4 

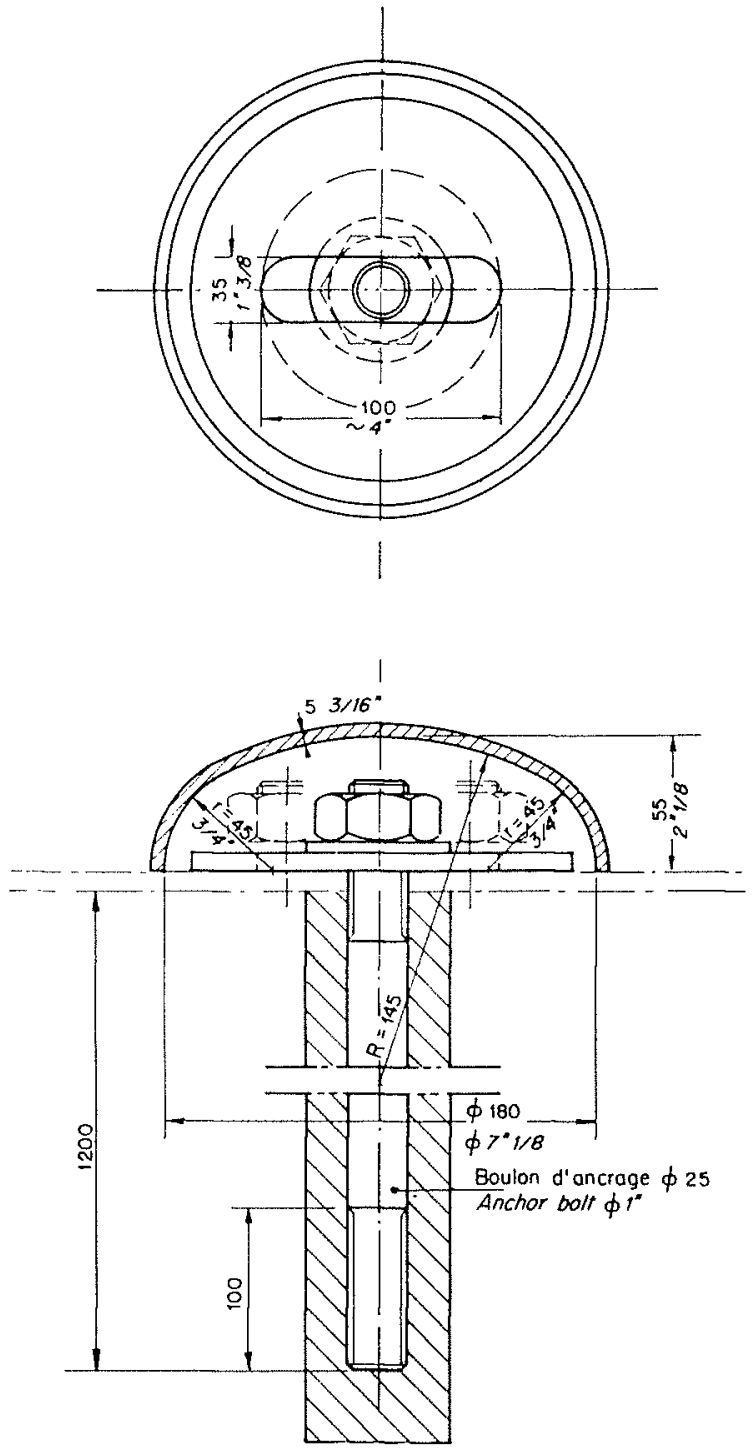

Fia. 5

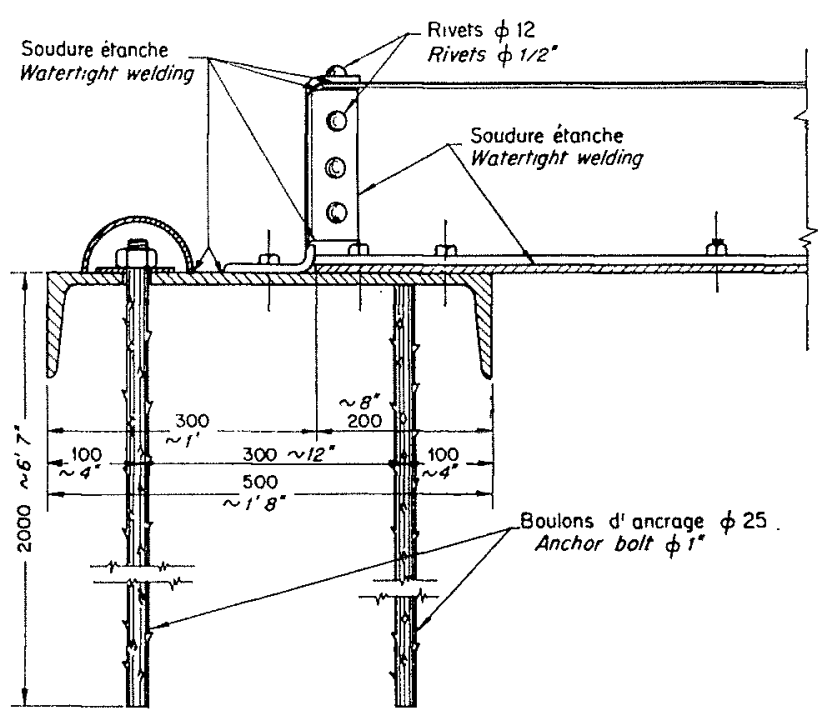

FIG. 6
$1,250 \mathrm{~m}$ de longueur; les trous pratiqués dans les tôles sont largement dimensionnés pour les dilatations prévues et sont couverts par des rondelles de grand diamètre convenablement serrées par l'écrou. Pour en assurer l'étanchéité, chaque tête de scellement est recouverte d'une coupole en tôle emboutie dont tout le pourtour est soigneusement soudé, comme on peut constater sur la figure 5 .

A son bord supérieur, le voile s'engage sous un masque, recourbé en brise-vagues et abritant l'extrémité du voile contre les paquets d'eau dus aux vagues, tout en maintenant constant l'aspect de l'ensemble du voile quelle que soit la température.

La liaison du voile métallique aux murs d'étanchéité en béton a constitué, bien entendu, le problème le plus délicat. La figure 6 montre la disposition figurant dans un premier projet. L'étanchéité était assurée par les ailes du fer U qui pénétraient dans le béton. La difficulté d'exécuter un bon bétonnage, les risques de décollements dus aux retraits, enfin l'impossibilité de trouver les poutrelles spécifiées, ont fait abandonner cette disposition. La figure 7 repré-

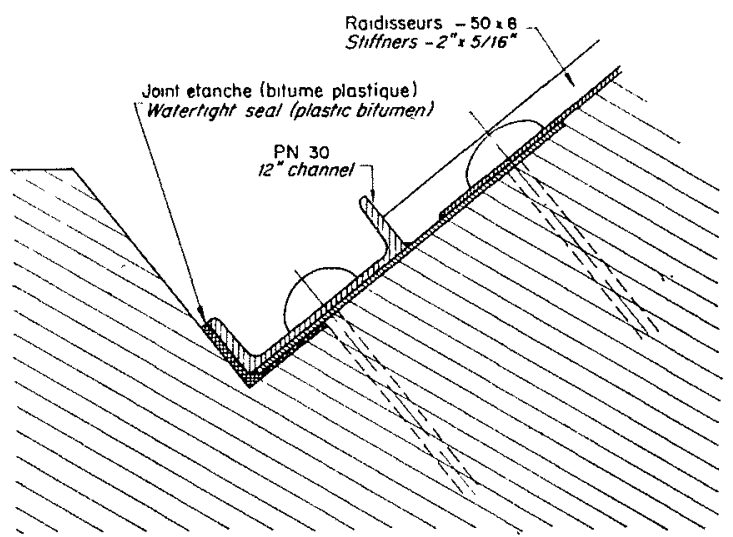

FIG. 7

sente le dispositif de liaison adopté pour la construction. Il est formé par un large plat de $600 \times 8$ raidi sur son bord extérieur par un fer U PN de $300 \mathrm{~mm}$, posé à plat et soudé. Le voile proprement dit repose également sur le plat et y est fixé par soudure. Cet ensemble est scellé au corps du barrage par une série de tiges analogues à celles qui maintiennent les tôles mais plus serrées et dont le trou ne permet pas de déplacement latéral. L'élément d'étanchéité est souple et est constitué par une émulsion bilumineuse armée de loile de jule, située sous l'extrémité extérieure du plat. Comme on le verra plus loin, ce système a montré des défauts à l'emploi et a dû être adapté en conséquence. 
Contre la corrosion, le voile a été abondamment revêtu de peinture bitumineuse Flintkote, tant sur l'endroit que sur l'envers et sur la couche de support en béton maigre, avant la pose.

L'envers des tôles a été traité sur chantier avant montage. Les tôles une fois mises à l'équerre, percées, munies de leur couvre-joint, étaient soigneusement nettoyées à la brosse métallique et au jet d'air, puis recouvertes de :

- une couche d'apprêt appliquée au pistolet, constituée d'une solution de bitume dans la térébenthine;

- une couche de protection de Flintkote $n^{\circ} 5$ appliquée à la brosse et à raison de $1 \mathrm{~kg} / \mathrm{m}^{2}$.

\section{D. - MONTAGE}

Dans un pareil ouvrage, le montage tient pratiquement lieu de fabrication et étant donné les tonnages impliqués, il a été jugé convenable d'exécuter sur un chantier le maximum d'opérations.

Les joints de contraction, raidisseurs, tiges de scellement, coupelles sont venus d'atelier tout préparés. Quant aux tôles, pour éviter des transports supplćmentaires et donner toute souplesse d'adaptation, elles ont été transportées directement des quais au chantier. Il est évident que le slockage et la préparation des tôles ont nécessité des installations assez importantes. Celles-ci sont représentées sur le plan figure 8 , ainsi que sur les photos A à I.

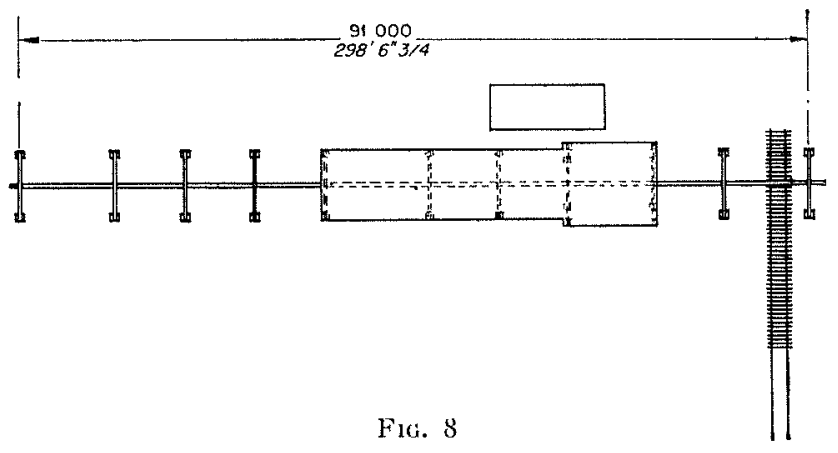

Le stockage et la préparation des tôles étaient disposés dans le prolongement l'un de l'autre et desservis par un monorail électrique suspendu à des chevalets en bois. Les tôles passaient successivement par le planage, traçage, mise à l'équerre par découpage ou chanfreinage simultané au chalumeau, perçage et soudage du couvre-joint, nettoyage et peinture, et aboutissaient sur un chariot double spécial.

Celui-ci était constitué par un chariot principal se déplaçant à la maín le long du couronnement sur une voie spécialement prévue à cet effet et portant un chariot-gigogne muni d'une armature sur laquelle les tôles étaient disposées. Cet ensemble secondaire étail orienté pour se mouvoir sur le plan du parement amont en $y$ roulant et comportait un dispositif de mise en place précise des tôles. Un treuil électrique spécial installé sur le chariot principal permettait d'atteindre n'importe quelle zone de la surface à revêtir. Une fois convenablement réglées à leur partie inférieure, les tôles y étaient retenues et déposées doucement sur le parement (pour ne pas abîmer la couche de protection).

Les opérations de montage se sont déroulées dans un ordre rigoureux en commençant par le traçage précis, sur le plan du parement, des axes des trous des ancrages, suivi de leur perforation et de leur scellement. On procédait ensuite à la mise en place des tôles par rangées horizontales successives, chaque tôle étant fixée par son ancrage et reliée par des points de soudure aux tôles voisines ainsi qu'aux joints de contraction et aux plats de raidissement. L'ensemble du voile fut ainsi mis en place et tenu par points de soudure avant qu'il ne fût procédé au soudage définitif.

Celui-ci donna lieu à des inconvénients imprévus et extrêmement difficiles à corriger. En effel, malgré toutes les précautions prises et les séquences essayées, les tôles ont présenté des déformations et gondolements visibles. Bien que n'influant pas sur la résistance de l'ouvrage, cela nuisait à son aspect et on chercha à atteindre une planéité parfaite.

Les recherches furent menées le plus rapidement possible et, en collaboration avec $M$. le professeur Albert Caquot, consulté à tilre d'Ingénieur-Conseil, d'excellents résultats furent obtenus. La séquence des soudures est :

a) Soudage des tôles entre elles, aux coins, sur environ $5 \mathrm{~cm}$;

b) Fixation des joints de contraction aux tôles par des points de soudure;

c) Soudage des joints de contraction aux tôles en partant du bas;

d) Soudage des tôles entre elles par deux soudures continues partant des joints de contraction et allant vers le milieu des tôles;

e) Soudage des barres sur champ. 


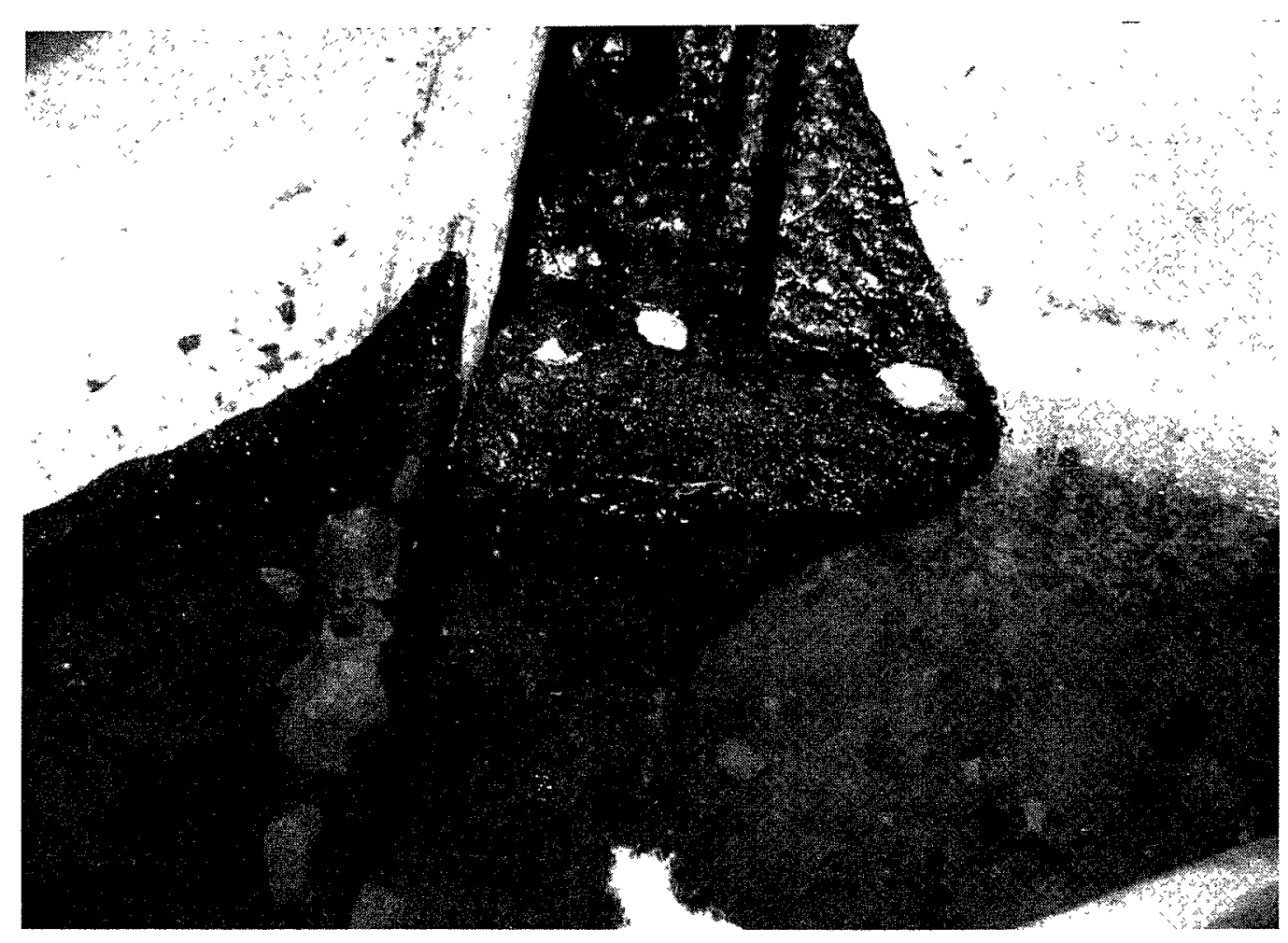

Pното A

Aspect des produits bitumineux d'étancheité, fondus par le soleil, se re-solidifiant dans l'eau de la retenue.

Pното B

Vue du soudage du voile étanche à partir du milieu montrant les soudeurs à l'wurre.

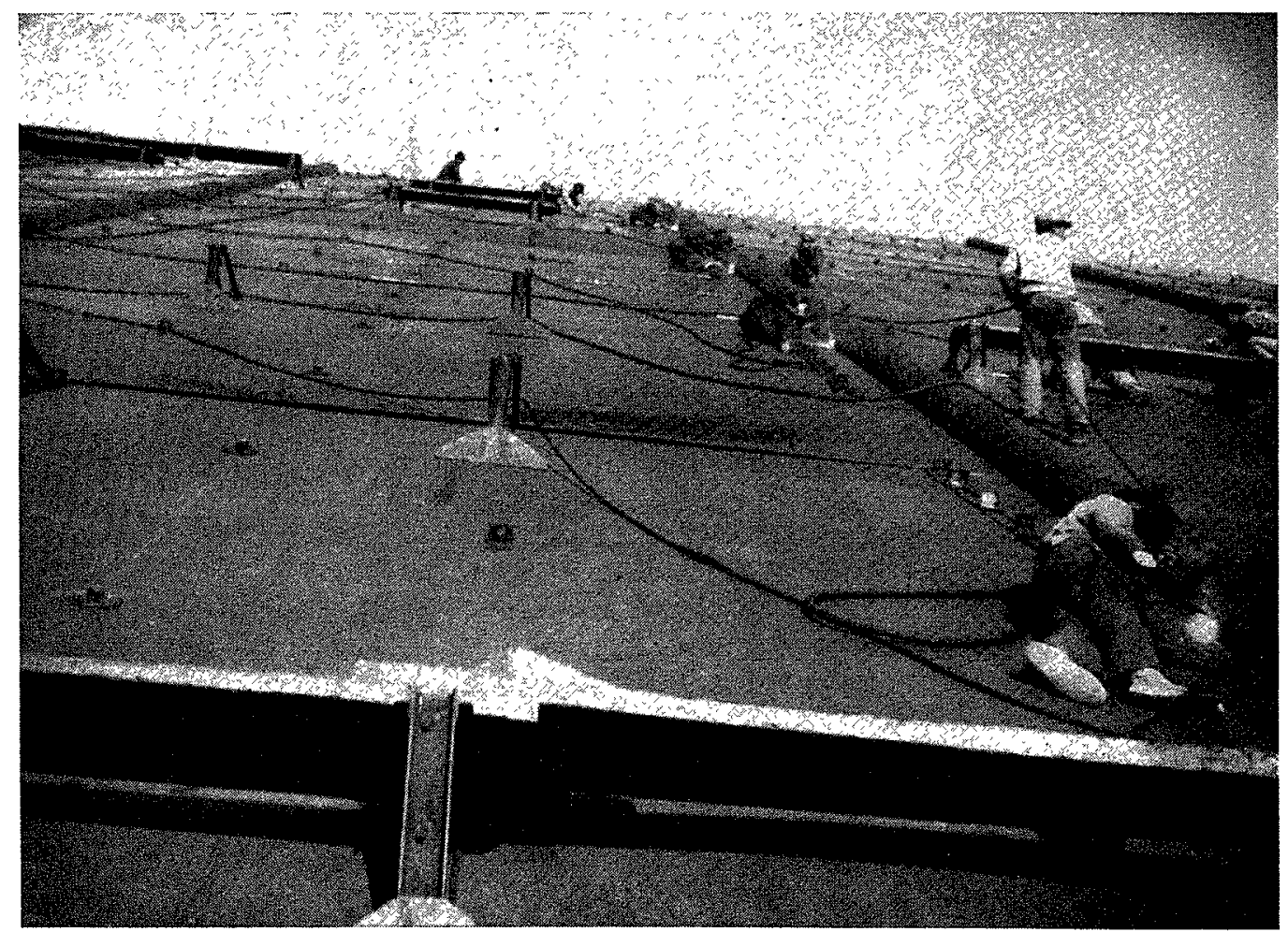




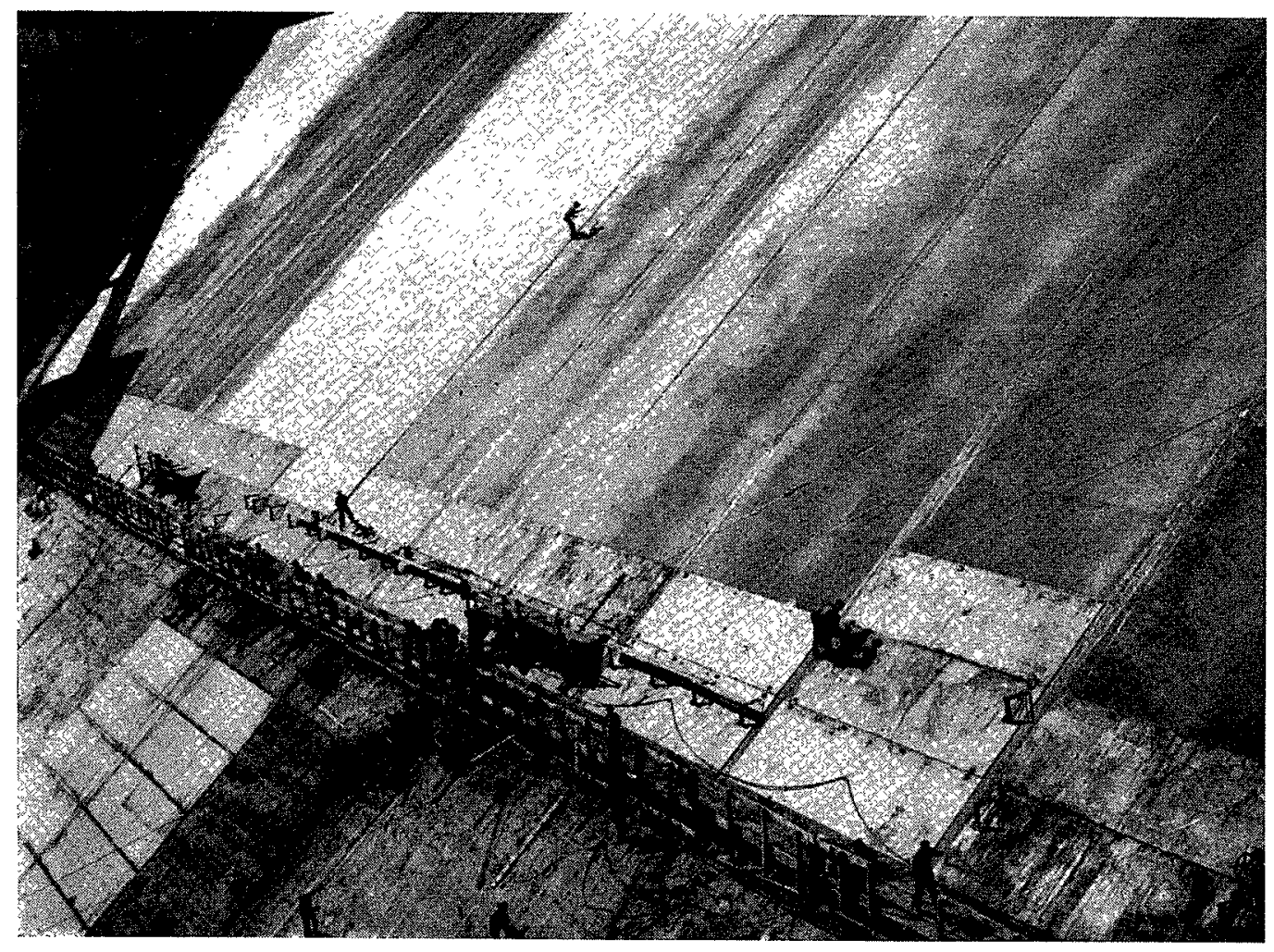

Photo C.

Débul de la pose des

tôles et

passerelle provisoire.

L'ouvrier, au milieu,

donne l'échelle.

On voit que la pente

a été

un facteur important.

Рното D

Fin du soudage: les joints de dilatation verticaux ont presque tous alteint le couronnement.

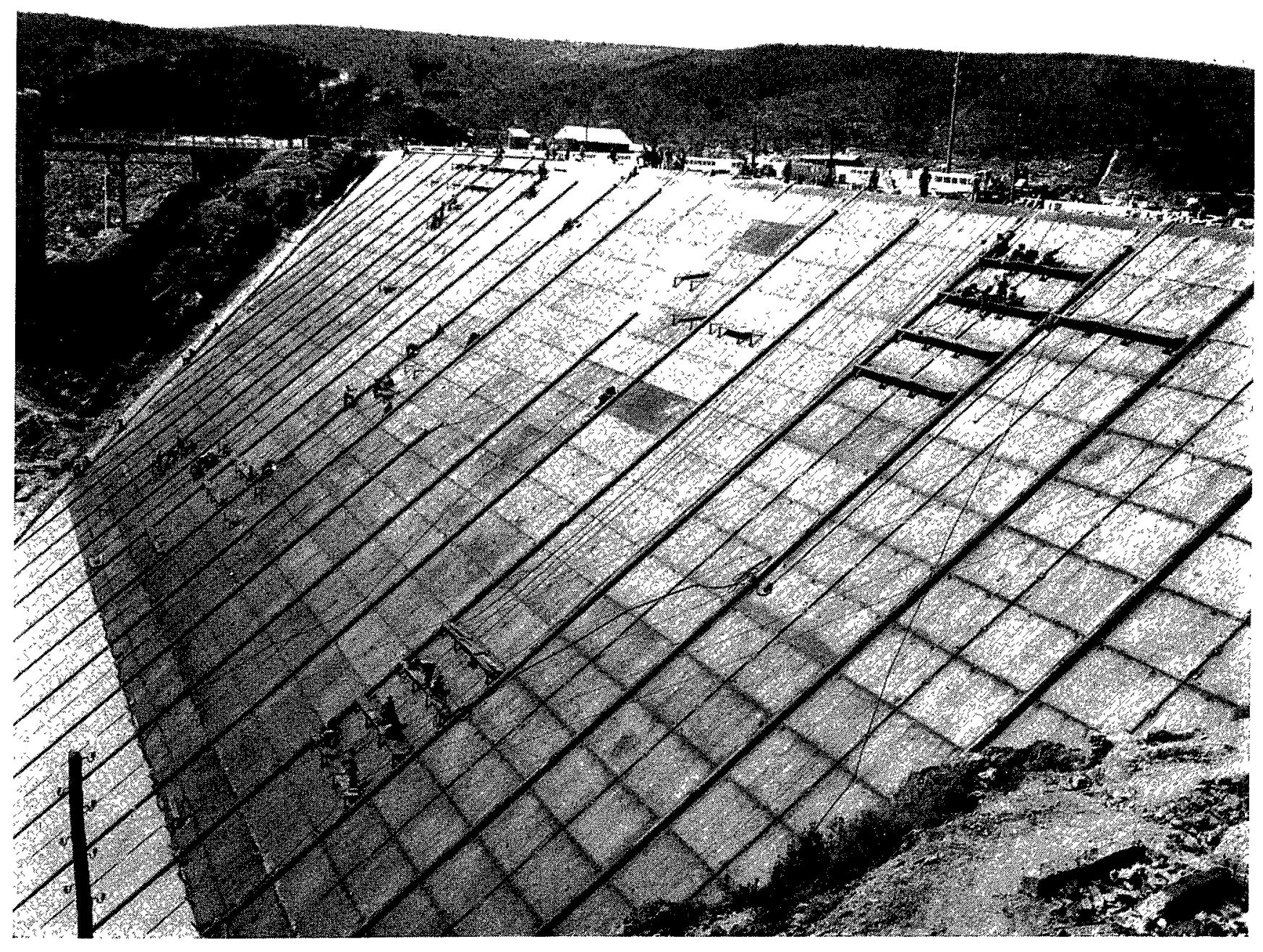




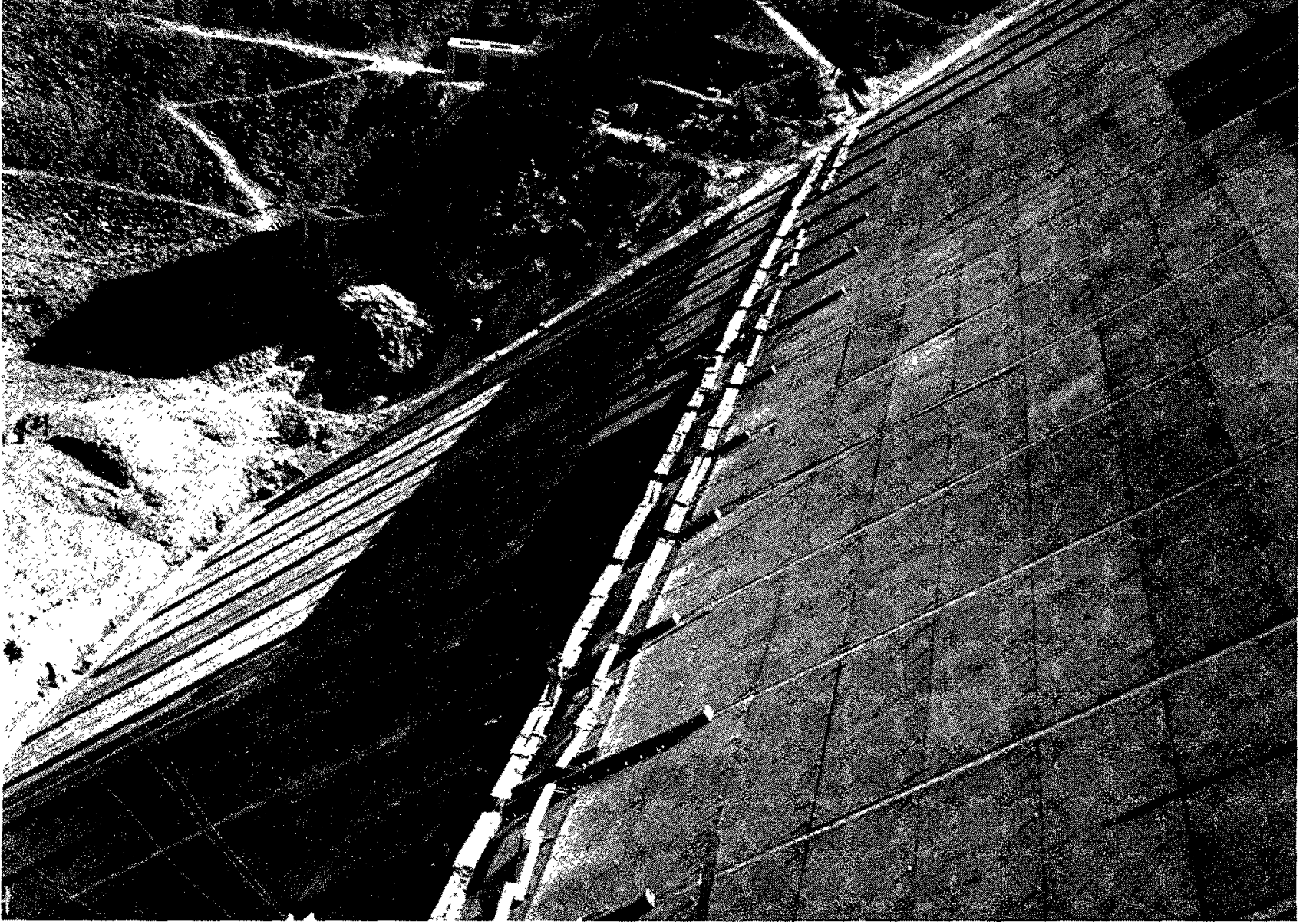

Pното E

Le voile etanche en cours de montage. La progression de la pose et du soudage des joints de dilatation depuis les bords vers le centre y est particulièrement visible.

Pното F

Vue d'ensemble des travaux de pose. A gauche les trois prises d'eau à des cotes différentes.
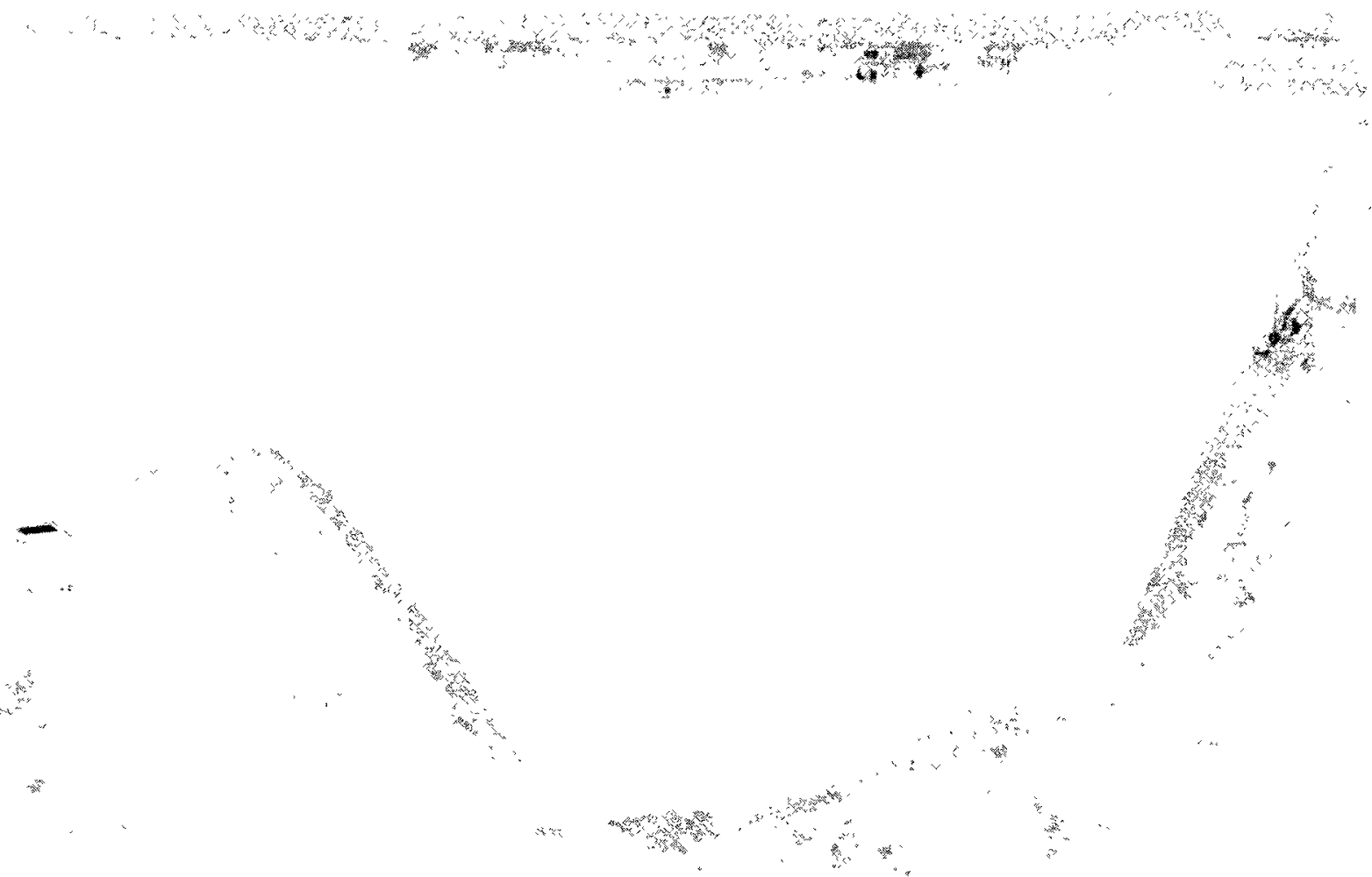
Pното G

Chariot-gigogne

replacé sur

le chariot principa!

après la

pose d'unc tôle.

Chariot-gigogne

sur le point

de poser une tôle.

Pното I

Le chariot-gigogne remonte après

la pose d'une tôle.
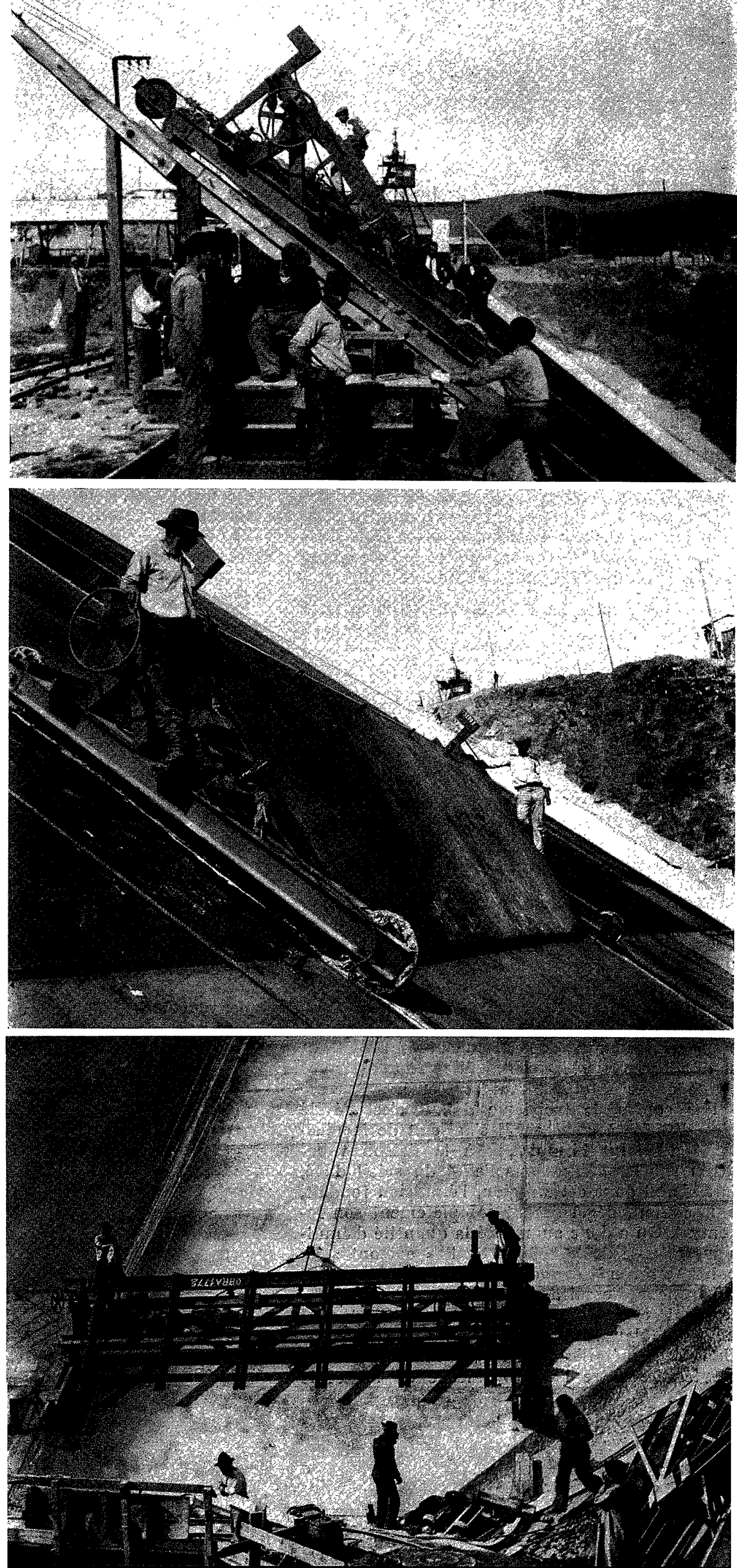
La peinture faite au moment du monlage (et mettant à part le revêtement de la couche-support de béton maigre) a consisté en une couche d'apprêt de peinture bitumineuse appliquée au pistolet, deux couches de protection de Flintkote $n^{\circ} 5$, appliquées à la brosse et à raison de $2 \mathrm{~kg} / \mathrm{m}^{2}$, el enfin un laitier de chaux mélangé ( 2 mesures) à du Flintkole (1 mesure) (mélange préparé sur place avec de l'eau salée), à raison de $2 \mathrm{~kg} / \mathrm{m}^{2}$.

A remarquer que la peinture, forcément appliquée à l'avance, a gêné les opérations de soudage, car la chaleur des électrodes causait des dégagements gazeux désagréables pour les opérateurs (bien qu'inoffensifs) et produisait des soufflures et défauts dans les cordons: d'où l'obligation fréquente de buriner et reprendre certaines soudures.

Les opérations de montage ont été un peu compliquées par deux impositions, toutes deux dues à l'urgence qu'il y avait de mettre le barrage en eau. La première difficulté, commune à de nom- breux chantiers, a été la poursuite simultanée des travaux de construction métallique et de génie civil. Ainsi, toutes les tôles ont été placées avant que la soudure définitive ne commence, permettant le démontage de la voie du chariot, l'achèvement de la route et de la balustrade du couronnement. Ainsi également, il a fallu fixer provisoirement la cote inférieure de dépôt des tôles aux environs de 29; une série de poutrelles I ont été encastrées dans le parement et ont soutenu l'ensemble du voile jusqu'à ce que la partie inférieure du parement ait été rendue libre pour que les travaux de construction du voile se poursuivent. La deuxième difficulté a été la vitesse à laquelle il a fallu travailler une fois les principales décisions prises; jusqu'à 24 soudeurs ont été utilisés simultanément, la moyenne de consommation d'électrodes par soudeur ayant atteint 22 électrodes/heure. Enfin il faut mentionner les très nombreuses éprouvettes de soudure exécutées et les excellents résultats obtenus.

\section{E. - MISE EN SERVICE ET TENUE DU VOILE}

La mise en service du voile n'a donné lieu à aucun incident. Les fuites ont été extrêmement réduites. Toutefois, au premier vidage de la retenue, on a constaté trois défauts.

Le premier, purement local, dû̀ à un matériau de mauvaise qualité, a été la rupture cristalline d'un des U PN 300 de la ceinture de liaison voilebéton. La réparation n'a pas posé de problèmes.

Le deuxième provenait d'un alignement déficient de deux joints de construction, dû à la hâte avec laquelle le montage avait été effectué. La soudure avait bien obturé l'interstice, mais, au boul d'un certain temps, le jeu des contractions et dilatations surcessives avait fini par détruire ce dépôt de soudure, mal supporté.

Par contre, le troisième défaut s'est révélé plus difficile à corriger. Il s'agissait de la plasticité du joint bitumineux à la liaison voilebéton sur les côtés. Sous l'effet du soleil d'été, fort chaud dans cette région, l'émulsion fondait, puis coulait, dégarnissant complètement son logement. On a donc augmenté la quantité d'émulsion et tenté de la retenir en l' « armant» de petits fers ronds soudés au laminé U PN (fig. 9). Un tronçon d'essai a montré que ces pointes retenaient effectivement l'émulsion bitumineuse mais que leur influence n'était pas suffisante. Une toile métallique à larges mailles a donc été tendue entre l'aile intérieure de l'U et le béton et la mesure étendue à l'ensemble du barrage. Cette solution s'est révélée parfaitement satisfaisante. Pendant la dernière année de mesures, le débit total de fuite du barrage a été nul.

En conclusion, nous pouvons constater que les voiles étanches métalliques, bien qu'étant d'une technique particulière d'exécution, offrent toutes garanties d'étanchéité et de résistance. De plus, appliqués en connaissance de cause, ils peuvent entraîner de substantielles économies tant par leur prix de premier établissement que

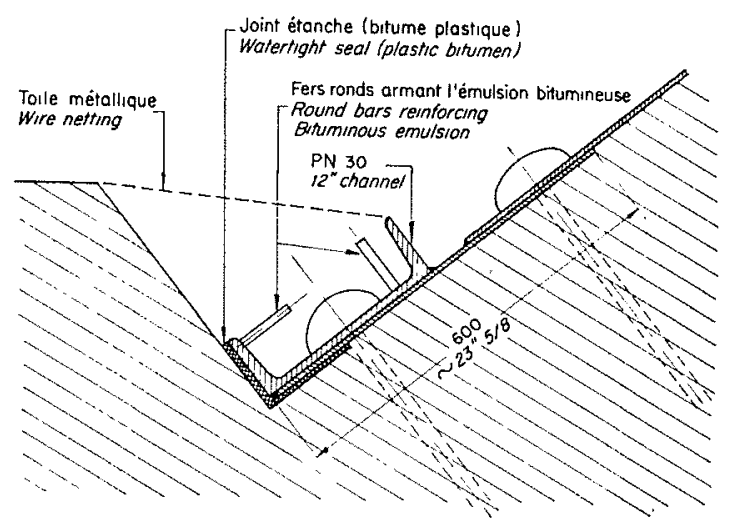

FIG, 9 
par la rapidité d'exécution. Le succès de celui du barrage Salazar en parait une éclatante confirmation.

Nous ne voudrions pas terminer sans rendre hommage à notre camarade Jean Bernaux, ingénieur des Arts et Manufactures, chef de Section aux Sociedades Reunidas de Fabricacões Metálicas adjudicataires du voile étanche. C'est, en effet, lui, en tanl que chargé de la construction et du montage du voile, ayant dû le mettre au point et surmonter successivement les difficultés qui se présentaient, qui aurait normalement écrit cet article, d'ailleurs avec une compétence bien plus grande que celle de l'auteur, si la maladie ne l'avait emporté aussitôt l'ouvrage terminé.

\section{BIBLIOGRAPHIE}

1" Barragens Salazar (Pego do Altar) et Trigo de Morais (Vale de Gaio) de obra de rega do Vale do Sado, communication des ingénieurs António Trigo DE Monals et Armando Palma Carnos, Quatrieme Congrès des Grands Barrages.

2" Metal-faced gravel dam being built in New Mexico, H.P. Bunger, Engineering News Record, October $26,1933$.

$3^{\circ}$ Second steel-faced dam built for Colorado Springs, Engineering News Record, Oct. 29, 1936.
4" Steel-facings for dams, par R. T. Logeman, Civil Engineering, Jan. 1939.

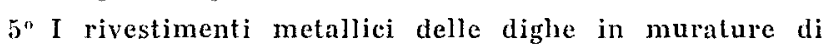
pietrame del lago del Diavolo e del Gabiet, par Dott. Ing. Giulio Gentme, Energia Elettrica, Feb. 1936.

6" Il rivestimento metallico della diga in pietrame a secco sul fiume Khram (Georgia-Transcaucasia), par Dott. Ing. Claudio Marcello, Energia Elettrica, Feb. 1936. 